\title{
Linac Sector 21-30 'QE' Quadrupole Magnet Excitation in LCLS Operation*
}

\author{
P. Emma \\ Dec. 10, 2006
}

\section{Introduction}

The existing SLAC linac quadrupole magnets, described as the 'QE' quadrupoles, are designed for excitation currents up to 200 A with length-integrated gradients up to $100 \mathrm{kG}$ needed to focus up to $50-\mathrm{GeV}$ electron or positron beams. The eight ' $\mathrm{QE}$ ' magnets within a linac sector are powered in series on a single 200-A bulk power supply, and each magnet in sectors 21-29 also includes a 20-A booster supply (adds 0-20 A to each magnet) for gradient tapering within the sector. Sector 30 is different again with individual 200-A power supplies per magnet. The LCLS will use these same quadrupole magnets, mostly in their existing locations and with their present power supplies, but for an electron energy range of $0.25-14 \mathrm{GeV}$. This much lower beam energy will require good power supply regulation $(\leq 0.05 \%$ rms for $f>0.5 \mathrm{~Hz})[1]$ and accurate field excitation at much lower currents, as low as a few amperes. The present (pre-2006) gradient-to-current polynomials (IVBU) in the SLC database are based on magnetic measurements at excitation currents above $20 \mathrm{~A}$, and are not accurate in extrapolation below about $10 \mathrm{~A}$. For this reason the IVBU polynomials for all 'QE' quadrupoles in sectors 21-30 will be updated in 2007 for LCLS operation. Magnets beyond Q21601 will be updated with a new generic polynomial (with polarity considerations) based on magnetic measurements from zero current all the way up to $200 \mathrm{~A}$, with additional data taken in the 0-20 A range for accuracy in LCLS mode (see Fig. 1). In addition, the first five LCLS 'QE' magnets (Q21201, Q21301, Q21401, Q21501, and Q21601), most of which run at the lowest currents of $<3 \mathrm{~A}$, have been individually measured and fitted with a specific polynomial to meet their special settings in LCLS mode. All changes will continue to fully support 30-50 GeV linac operations. This note describes these changes and the method used to arrive at this solution. Regulation tests have already been run on both the bulk and booster power supplies which demonstrate a regulation level below

Work supported in part by the DOE Contract DE-AC02-76SF00515.

This work was performed in support of the LCLS project at SLAC. 
$0.05 \%$ rms [1]. Table 2 lists a summary of the gradient-to-current polynomials needed in 2007 for all 'QE' magnets from sector-21 to 30.

\section{Gradient Sensitivities and Generic Polynomials}

The gradient sensitivities, $\Delta G / G$, for the first seven LCLS ' $\mathrm{QE}$ ' magnets are listed below in Table 1. Each error will cause a beta-mismatch amplitude change, $\Delta \zeta$, of $1 \%$.

$$
\Delta \zeta=\frac{1}{2} \beta^{2} k^{2} L^{2}\left(\frac{\Delta G}{G}\right)^{2}
$$

(The 'QE' magnets have an effective magnetic length of $L=0.1068 \mathrm{~m}$.) The table shows that, except for Q21201, 3-4\% gradient errors are not a major problem. Beyond Q21601 the gradient sensitivities (i.e., $\Delta \zeta \approx 1 \%$ ) are constant at about $4 \%$ for almost all ' $Q E$ ' magnets (except a few in the BC2 region, which need special attention in any case).

Table 1. Sector-21 ' $\mathrm{QE}$ ' quadrupole magnet settings and sensitivities, $\Delta G / G$. Here $\beta_{\max }$ is the maximum beta function in either $x$ or $y$.

\begin{tabular}{|l|c|c|c|c|c|c|c|c|}
\hline $\begin{array}{l}\text { Quadrupole } \\
\text { location }\end{array}$ & $\begin{array}{c}\text { old* serial } \\
\text { number }\end{array}$ & $\begin{array}{c}\text { new* serial } \\
\text { number }\end{array}$ & $\begin{array}{c}\boldsymbol{k}_{\text {nom }} \\
\left(\mathbf{1} / \mathbf{m}^{2}\right)\end{array}$ & $\begin{array}{c}\mathcal{G} \boldsymbol{G} \mathbf{l} \\
\mathbf{( k G )}\end{array}$ & $\begin{array}{c}|\boldsymbol{I}| \\
\mathbf{( A )}\end{array}$ & $\begin{array}{c}\boldsymbol{\beta}_{\max } \\
\mathbf{( m )}\end{array}$ & $\begin{array}{c}|\Delta \boldsymbol{G} / \boldsymbol{G}| \\
(\mathbf{\%})\end{array}$ & $\begin{array}{c}\boldsymbol{E} \\
(\mathbf{G e V})\end{array}$ \\
\hline Q21201 & QE-125 & QE-072 & -9.3 & -8.3 & 15 & 12 & 1.2 & 0.25 \\
\hline Q21301 & QE-137 & QE-004 & -0.13 & -0.12 & 0 & 5 & 200 & 0.25 \\
\hline Q21401 & QE-150 & QE-002 & +1.05 & +1.4 & 2.2 & 46 & 3.0 & 0.38 \\
\hline Q21501 & QE-169 & QE-125 & -0.83 & -1.5 & 2.4 & 44 & 3.5 & 0.52 \\
\hline Q21601 & QE-185 & QE-137 & +0.71 & +1.7 & 2.8 & 45 & 4.2 & 0.67 \\
\hline Q21801 & $?$ & same & -0.71 & -2.1 & 3.5 & 44 & 4.2 & 0.82 \\
\hline
\end{tabular}

* "Old" and "new" refer to magnet serial numbers before and after, respectively, LCLS installation beginning in Aug. 2006 (note, sometimes the "Q" does not appear on the magnet).

With 80 'QE' magnets in the LCLS linac, it is not practical to remove and re-measure them all. So in order to determine how many of the early ' $Q E$ ' quadrupoles to remeasure, five magnets (serial numbers: QE-072, QE-004, QE-002, QE-125, QE-137) were magnetically measured in an experiment. Figure 1 shows the excitation current vs. length-integrated gradient for each magnet, all fitted simultaneously to one $5^{\text {th }}$-order polynomial. The fit excludes QE-125 (see below), which is about 2.5\% different than the other magnets for no clear reason, and QE-072 was measured twice over a 6-month period. Measurements are taken more densely in the 0-20 A region to better represent LCLS excitation levels, while measurements are also taken up to $200 \mathrm{~A}$ in 20-A steps. The coefficients are listed on the plot ( $p_{0}$ through $p_{5}$ ) assuming the following polynomial, 


$$
I=\sum_{i=0}^{5} p_{i}\left(\int G d L\right)^{i}
$$

where $\int G d L$ is the length-integrated field gradient, in $\mathrm{kG}$, and $I$ is the excitation current, in Amperes, necessary to achieve that gradient.

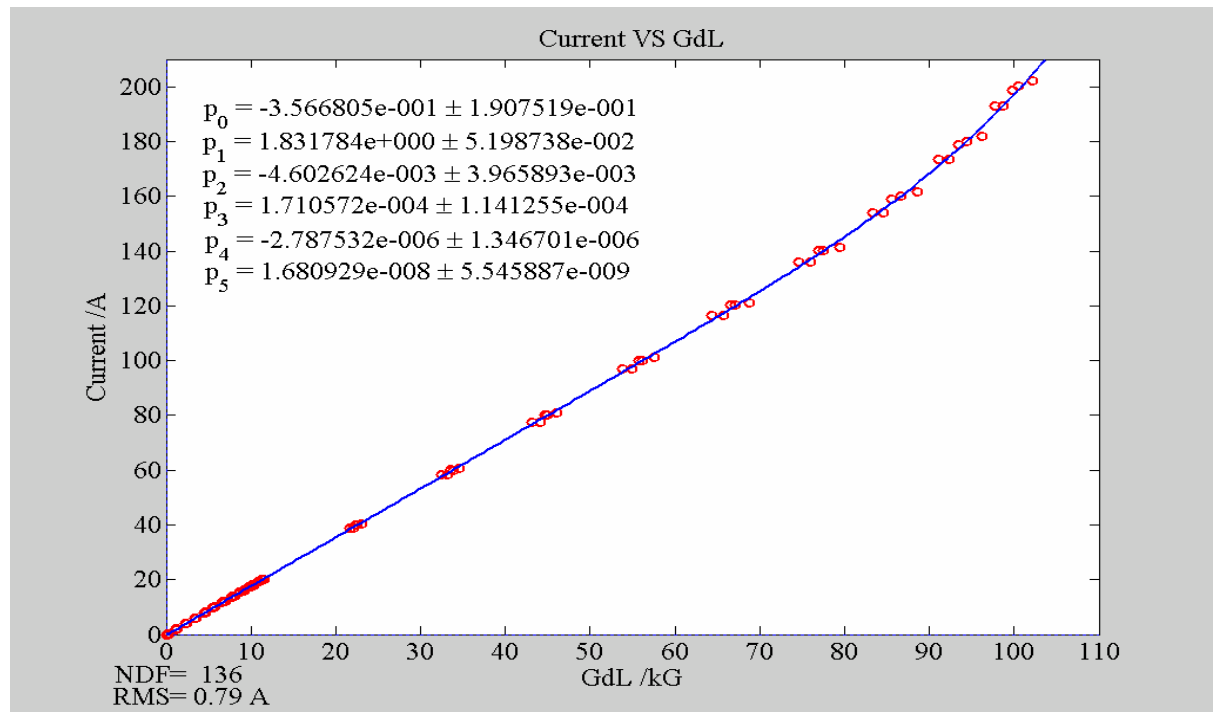

Figure 1. Excitation current vs. length-integrated gradient for each of the magnets: QE072 (twice), $-002,-004,-137$. One $5^{\text {th }}$-order polynomial is fit to all five measurements (four different magnets) simultaneously. This curve shows a polynomial where $+\int G d L$ produces $+I$ for even-numbered locations (i.e., Q2n201, 401, 601, 801). For the oddnumbered locations (i.e., Q2n301, 501, 701, 901) these odd polynomial coefficients ( $p_{1}$, $\left.p_{3}, p_{5}\right)$ must flip sign (see Table 2).

The relative deviations from this generic polynomial are shown in Fig. 2a (0-200 A scale) and Fig. 2b (tighter LCLS scales). The relative gradient errors induced by using one generic polynomial depend on the excitation current. The large errors below about $3 \mathrm{~A}$ are avoided by using a specific polynomial for these cases, rather than the generic polynomial. Above $3 \mathrm{~A}$, the relative errors drop to $<2 \%$. From the excitation current column of Table 1 (i.e., the column labeled: “|I|”), all linac 'QE' quadrupoles beyond Q21601 run at currents above 3 A. From this consideration, we use specific polynomials for each ' $Q E$ ' magnet up to and including Q21601, and use a generic polynomial for all magnets beyond Q21601. (Note that the odd polynomial coefficients $\left(p_{1}, p_{3}, p_{5}\right)$ must flip sign when applied to odd-numbered locations (i.e., Q2n301, 501, $701,901)$ so that the polarity is such that $-\int G d L$ produces $+I$ for these odd unit number quads). Only QE-125 does not fit the generic polynomial (at $I>3$ A) to within $\pm 1.5 \%$ (see magenta points with “ $x$ ” plotting symbol in Figs. 2a and 2b). As long as there are not many magnets like QE-125 in the unmeasured sections beyond Q21601, this should not be a problem. QE-125 has been individually measured and used as shown in Table 1. 


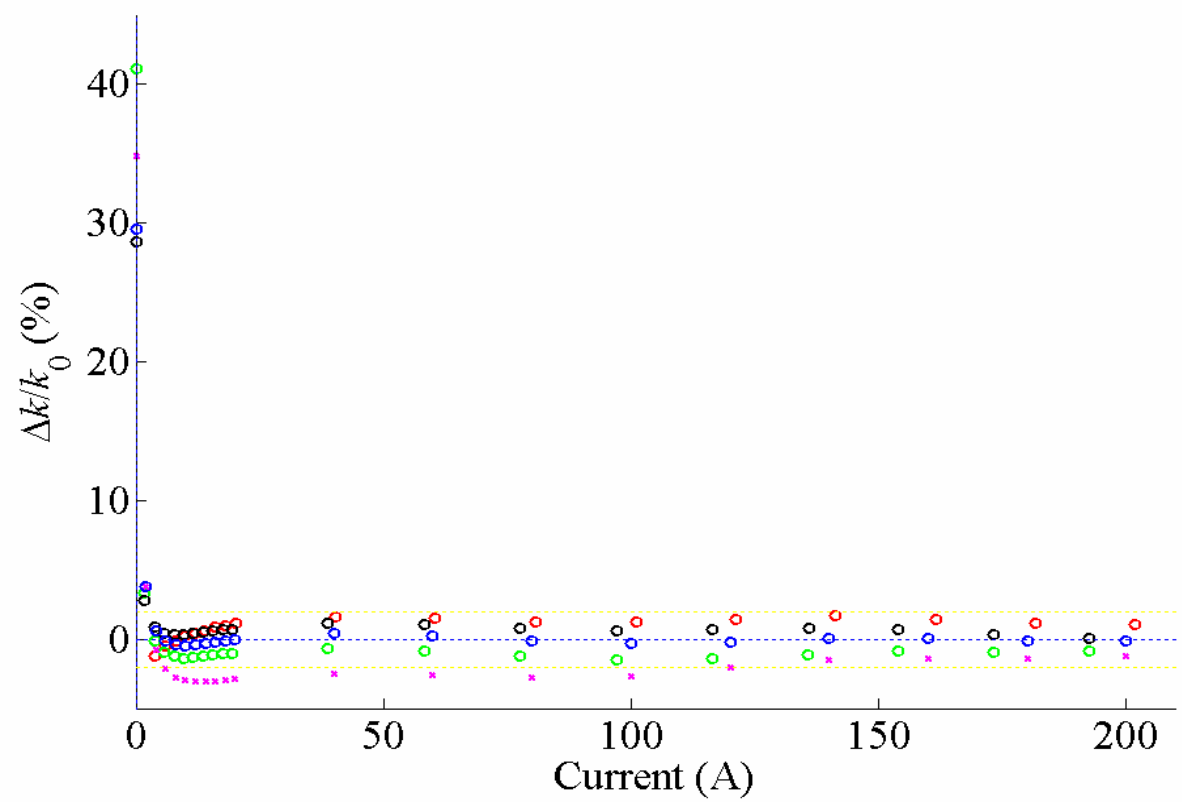

Figure 2a. Relative gradient errors $(\Delta k / k=\Delta G / G)$ for all four measured 'QE' quadruples with respect to the generic polynomial of Fig. 1. All ' $Q E$ ' quadrupoles above a 3-A level (i.e., beyond $Q 21601$ ) can be run with this generic polynomial with a gradient error $<2 \%$, unless they are anomalous magnets such as QE-125 (magenta “ $\times$ ” plotting symbol).

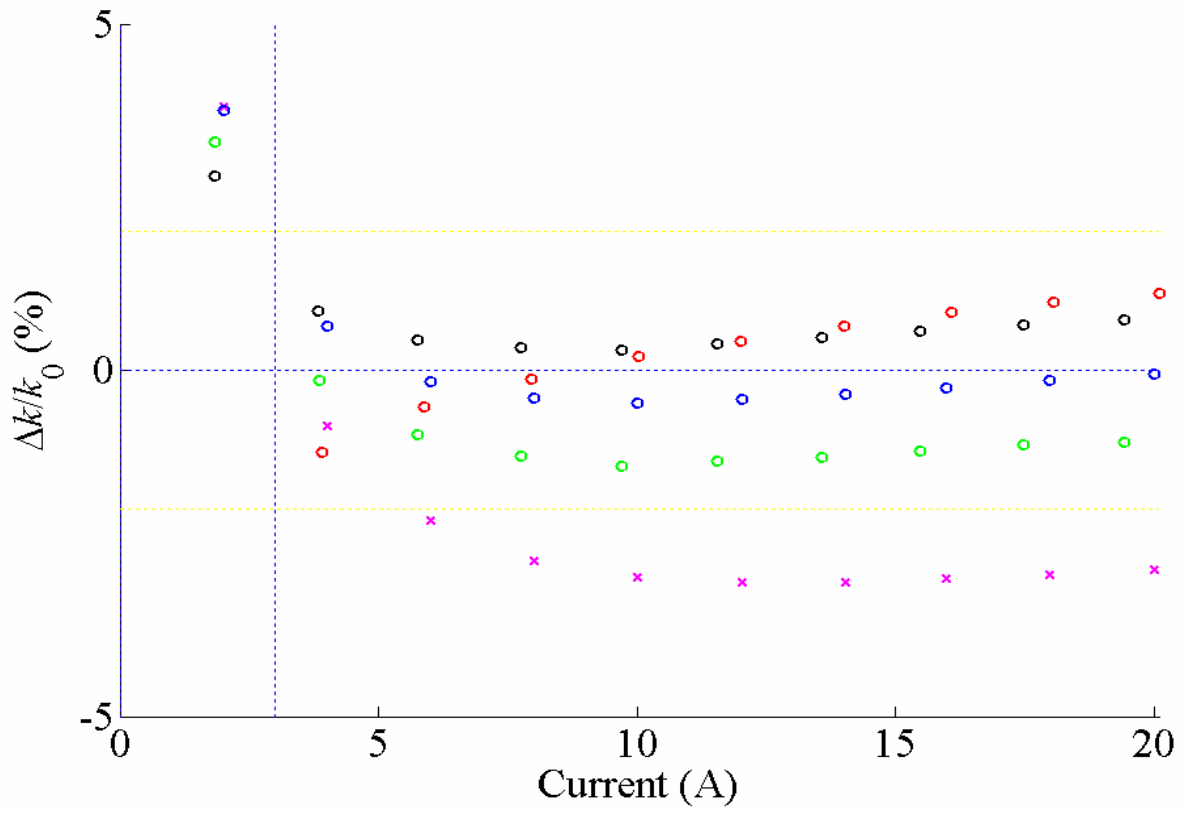

Figure 2b. Same as Fig 2a but with tighter (LCLS) vertical and horizontal scales. The magenta points with " $\times$ ” plotting symbols are from QE-125 and not included in the generic polynomial fit, so this $>2 \%$ error will not arise as long as other magnets are not anomalous like QE-125; an unknown issue at present, but not unique to LCLS. 


\section{Quadrupole Magnet Q21201}

The first 'QE' magnet in the LCLS beamline is Q21201. This magnet will be moved about 1.1 meters downstream from its pre-LCLS location (prior to Aug. 21, 2006). This magnet must run in a reversed polarity in LCLS mode (with respect to 'SLC' mode) and at a low current of about $-15 \mathrm{~A}$, so a reversing switch has been installed on its 20-A booster supply. The sector-21 bulk supply also has a disconnecting switch installed so that it provides no bulk current to any of the eight sector-21 'QE' magnets during LCLS operations. These eight will be excited only by their booster supplies in LCLS mode. The booster supply current readback for the Q21201 magnet will be tapped before the reversing switch so that the measured current is always positive. The field readback polarity is handled by switching from the IVBU database polynomial ('SLC' mode) to the IVBD polynomial (LCLS mode). The polynomial are identical, except that the odd coefficients have reversed signs (see Table 2). In 'SLC' mode $+\int G d L$ produces $+I$, while in LCLS mode $-\int G d L$ produces $+I$. (The BMAX sign is also flipped to negative in LCLS mode.) This treatment allows a mode switch-over, from 'SLC' to LCLS, without the need to re-boot (IPL) the LI21 microprocessor.

The standard cycle used for the Q21201 magnet requires it to be ramped (10 A/s) up to $+200 \mathrm{~A}$ for 10 seconds, then ramped down to zero where the polarity is switched and the bulk supply disconnected, and finally the magnet is set to -15 A using the boost supply. Fig. 3 shows the magnetic measurements made on magnet serial number QE-072 with a $5^{\text {th }}$-order polynomial fit.

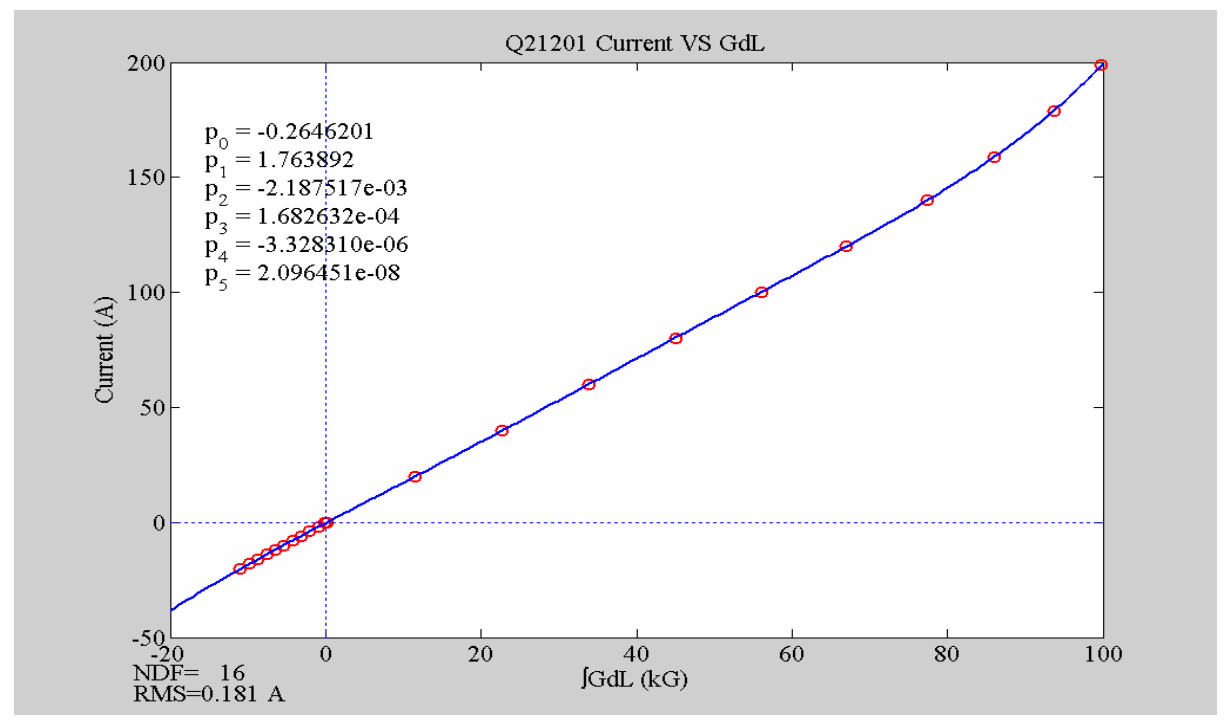

Figure 3. Magnet at Q21201 location (serial number QE-072). The magnet gets ramped up to $+200 \mathrm{~A}$ for 10 seconds, then down to zero, then reversed, and then ramped to $-15 \mathrm{~A}$. For LCLS mode, the odd polynomial coefficients get flipped in sign (using IVBD). This curve shows the polynomial in SLC mode (IVBU) where $+\int G d L$ produces $+I$. 


\section{Quadrupole Magnet Q21301}

The second 'QE' magnet in the LCLS beamline is Q21301. This magnet will be moved about 1.2 meters downstream from its pre-LCLS location (prior to Aug. 21, 2006). This magnet must run with zero excitation current in LCLS mode, with only its remnant field. A shorting switch is provided to guarantee zero current. The standard cycle used for this magnet requires it to be ramped $(10 \mathrm{~A} / \mathrm{s})$ up to $+200 \mathrm{~A}$ for 10 seconds, and then ramped down to zero where the bulk supply is disconnected and the shorting switch is set. The remnant field has been carefully measured and is included in the optical design of the LCLS. Fig. 4 shows the magnetic measurements made on magnet serial number QE-004 with a $5^{\text {th }}$-order polynomial fit.

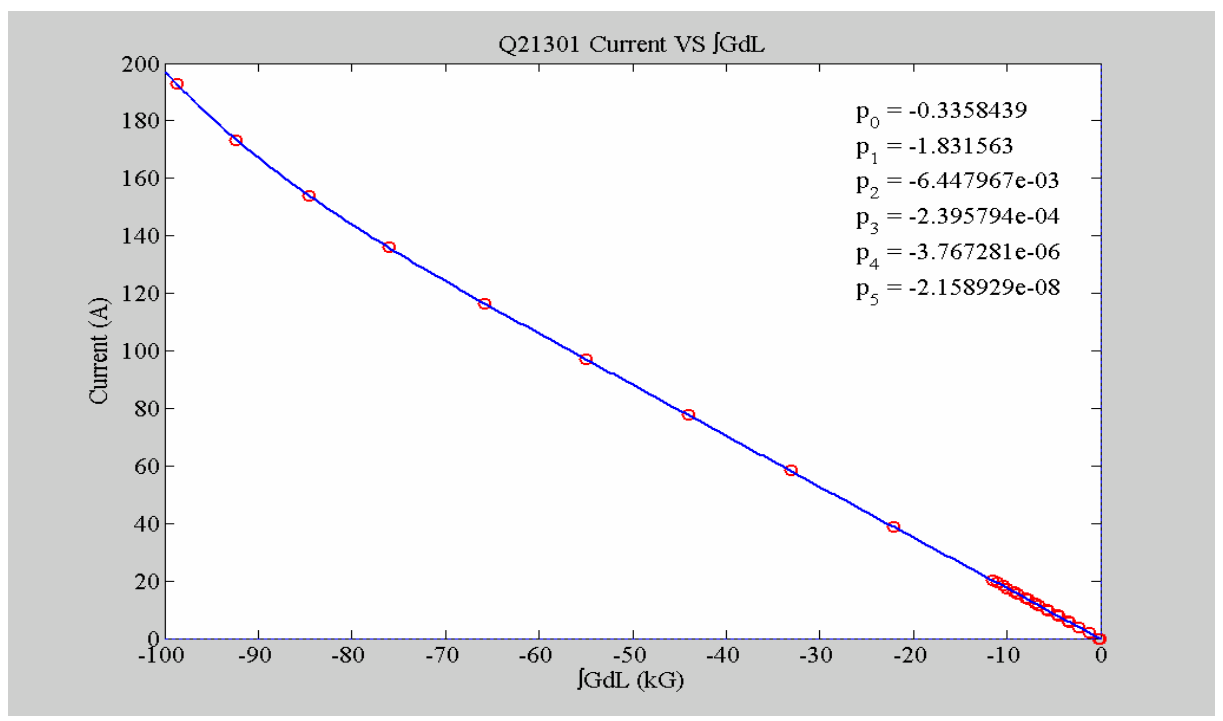

Figure 4. Magnet at Q21301 location (serial number QE-004). The magnet gets ramped up to +200 A for 10 seconds, then down to zero, and then shorted out with only its remnant field remaining. The polarity is such that $-\int G d L$ produces $+I$ for LCLS \& 'SLC'.

\section{Quadrupole Magnet Q21401}

The third 'QE' magnet in the LCLS beamline is Q21401. This magnet will not be moved. It will run in a low current state for LCLS mode, at about $2.2 \mathrm{~A}$. The standard cycle used for this magnet will require it to be ramped $(10 \mathrm{~A} / \mathrm{s})$ up to $+200 \mathrm{~A}$ for 10 seconds, then ramped down to zero for 10 seconds, where the bulk supply is disconnected and the booster supply is set at $+2.2 \mathrm{~A}$. The field at this low current has been carefully measured. Fig. 5 shows the magnetic measurements made on magnet serial number QE-002 with a $5^{\text {th }}$-order polynomial fit. 


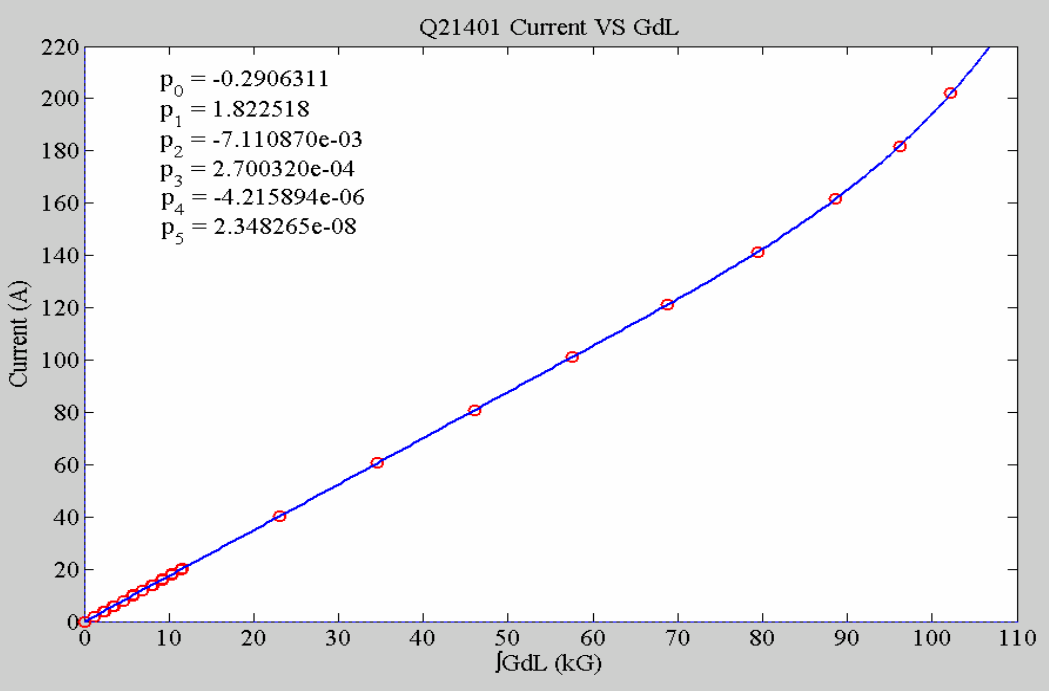

Figure 5. Magnet at Q21401 location (serial number QE-002). The polarity is such that $+\int G d L$ produces $+I$ for LCLS and 'SLC'.

\section{Quadrupole Magnet Q21501}

The fourth ' $\mathrm{QE}$ ' magnet in the LCLS beamline is Q21501. This magnet will not be moved. It will run in a low current state for LCLS mode, at about 2.4 A. The standard cycle will require it to be ramped $(10 \mathrm{~A} / \mathrm{s})$ up to $+200 \mathrm{~A}$ for 10 seconds, then down to zero for 10 seconds, where the bulk supply is disconnected and the booster supply is set at +2.4 A. The field at this low current has been carefully measured. Fig. 6 shows the magnetic measurements made on magnet QE-125 with a $5^{\text {th }}$-order polynomial fit.

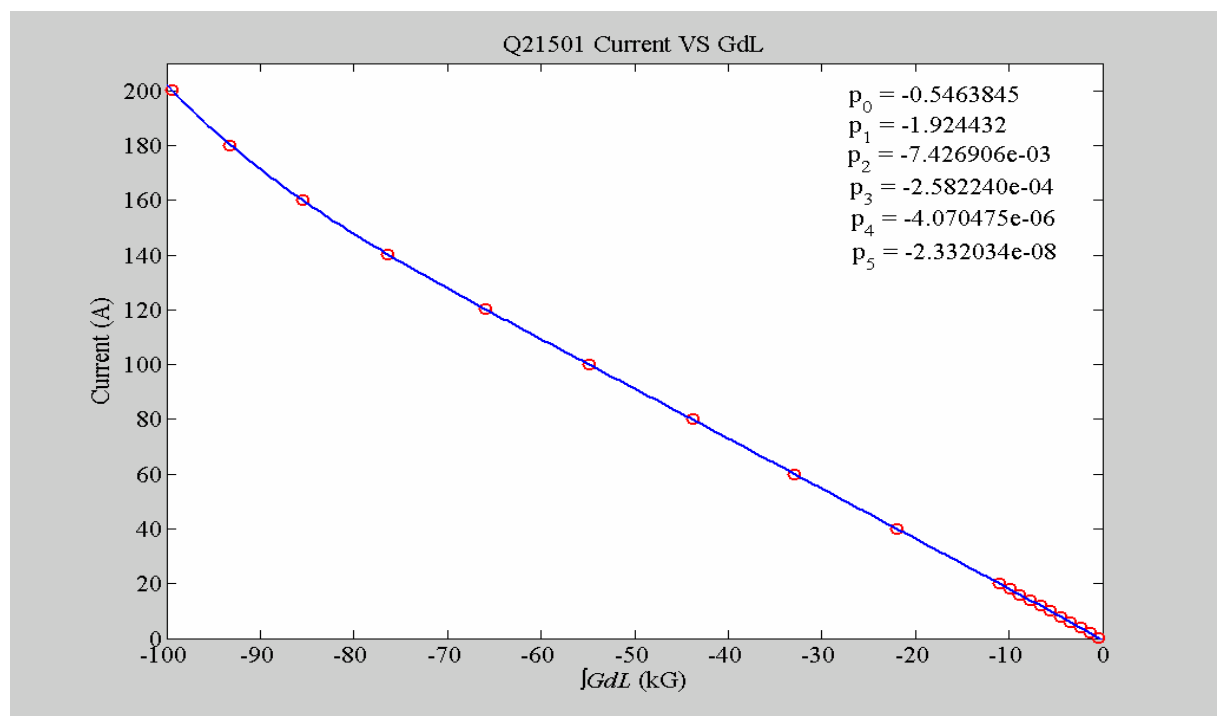

Figure 6. Magnet at Q21501 location (serial number QE-125). The polarity is such that $-\int G d L$ produces $+I$ for LCLS and 'SLC'. 


\section{Quadrupole Magnet Q21601}

The fifth 'QE' magnet in the LCLS beamline is Q21601. This magnet will not be moved. It will run in a low current state for LCLS mode, at about $2.8 \mathrm{~A}$. The standard cycle will require it to be ramped $(10 \mathrm{~A} / \mathrm{s})$ up to $+200 \mathrm{~A}$ for 10 seconds, then ramped down to zero for 10 seconds, where the bulk supply is disconnected and the booster supply is set to +2.8 A. The field at this low current has been carefully measured. Fig. 7 shows the magnetic measurements made on magnet QE-137 with a $5^{\text {th }}$-order polynomial fit.

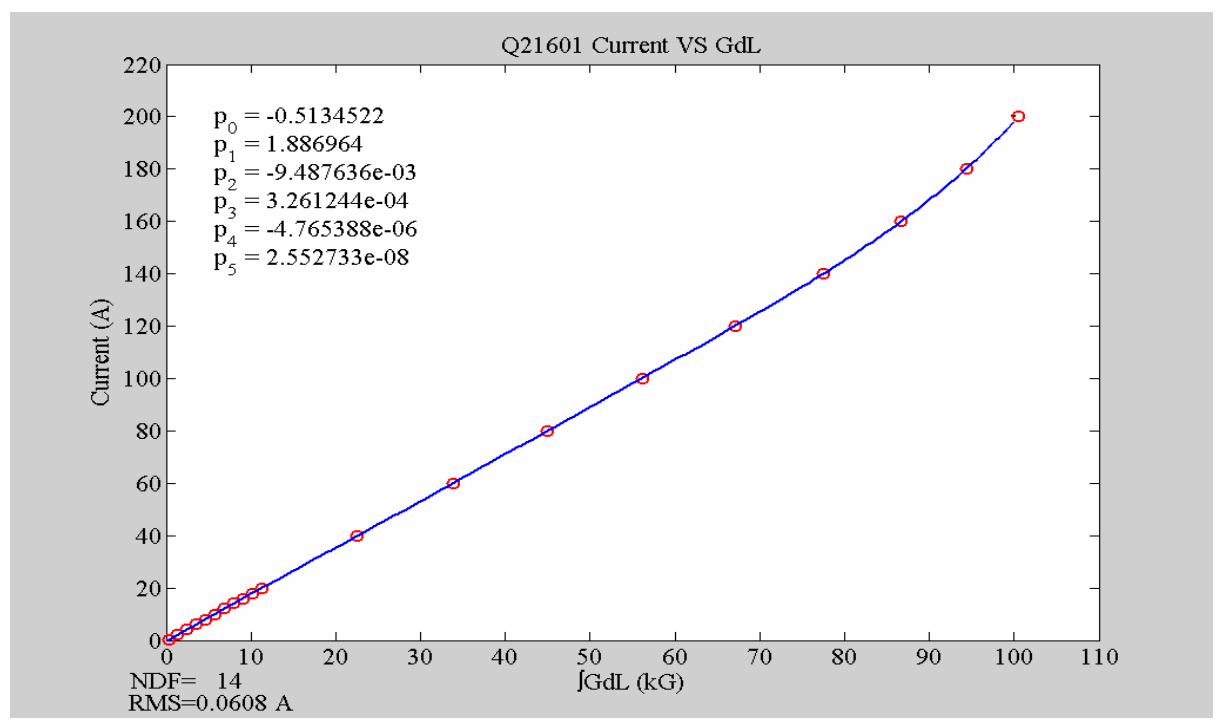

Figure 7. Magnet at Q21601 location (serial number QE-137). The polarity is such that $+\int G d L$ produces $+I$ for LCLS and 'SLC'.

\section{QE-Quadrupole Magnets Beyond Q21601}

The other 'QE' magnets in the LCLS beyond the Q21601 location are not moved (except Q24801 which is removed completely, and Q24701 and Q24901, both of which get doubled-up as two adjacent 'QE' magnets). The magnets beyond Q21601 will use the generic polynomial of Fig. 1. However, the odd polynomial coefficients $\left(p_{1}, p_{3}, p_{5}\right)$ must flip sign when applied to 'odd-numbered' locations (i.e., Q2n301, 501, 701, 901) so that the polarity is such that $-\int G d L$ produces $+I$ for these 'odd' unit numbered quads (see Table 2).

The LCLS generic polynomial is plotted (dashed line) in Fig. 8, along with the data from the pre-2006 sector 21-30 polynomials (all pre-2006 database polynomials are identical within a sector), and the QE-002, 004, 072, 125, and QE-137 polynomials. Figure 9 shows the absolute (top) and relative (bottom) current errors of these polynomials with respect to the LCLS generic polynomial. The large errors at $I<10$ A for the pre-2006 sector 21-30 data are due to the inaccurate old polynomials, which were based on data 
above $20 \mathrm{~A}$ only. The other errors are reduced to $<2 \%$ (above $3 \mathrm{~A}$ ) by using specific polynomials for Q21201 through Q21601, assuming QE-125 is an isolated event. (This cannot be fully confirmed unless all 75 remaining 'QE' magnets are removed and measured.)

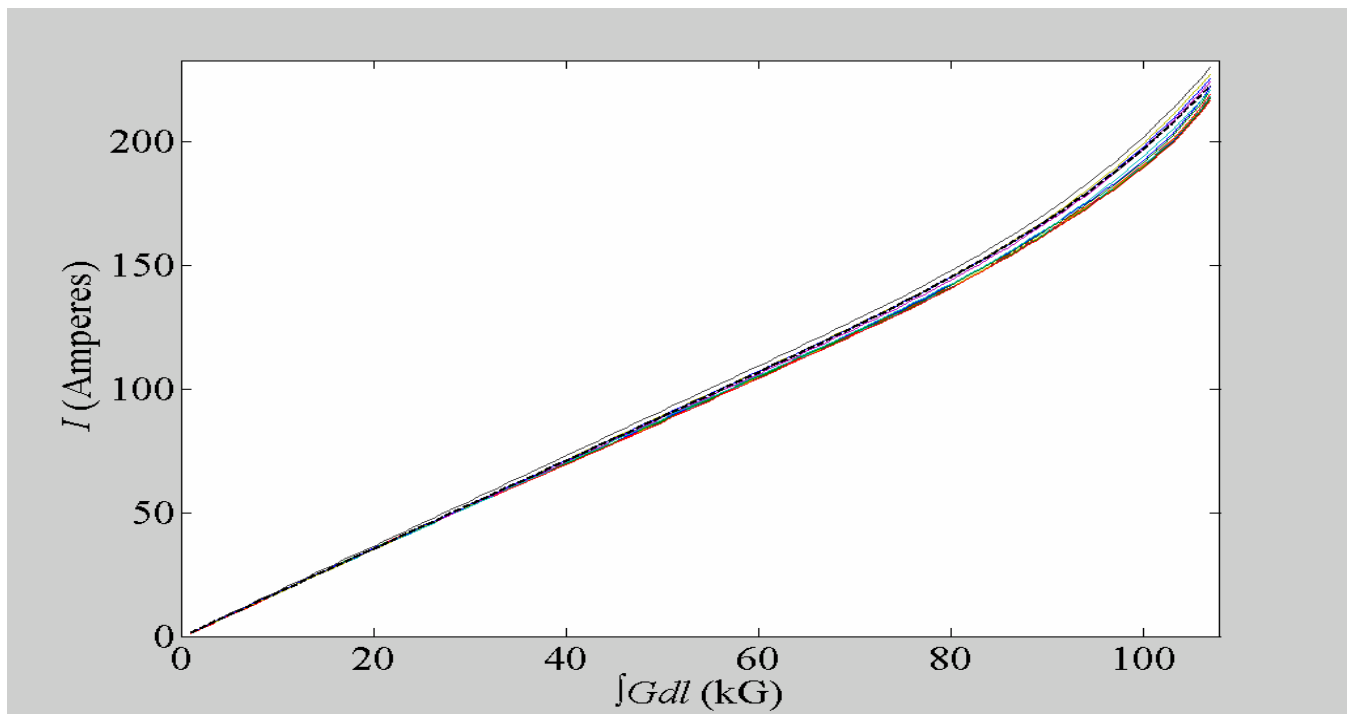

Figure 8. Pre-2006 SLC database polynomials from sectors 21-30, QE-002, 004, 072, 125, 137 polynomials, and the LCLS generic polynomial (dashed).

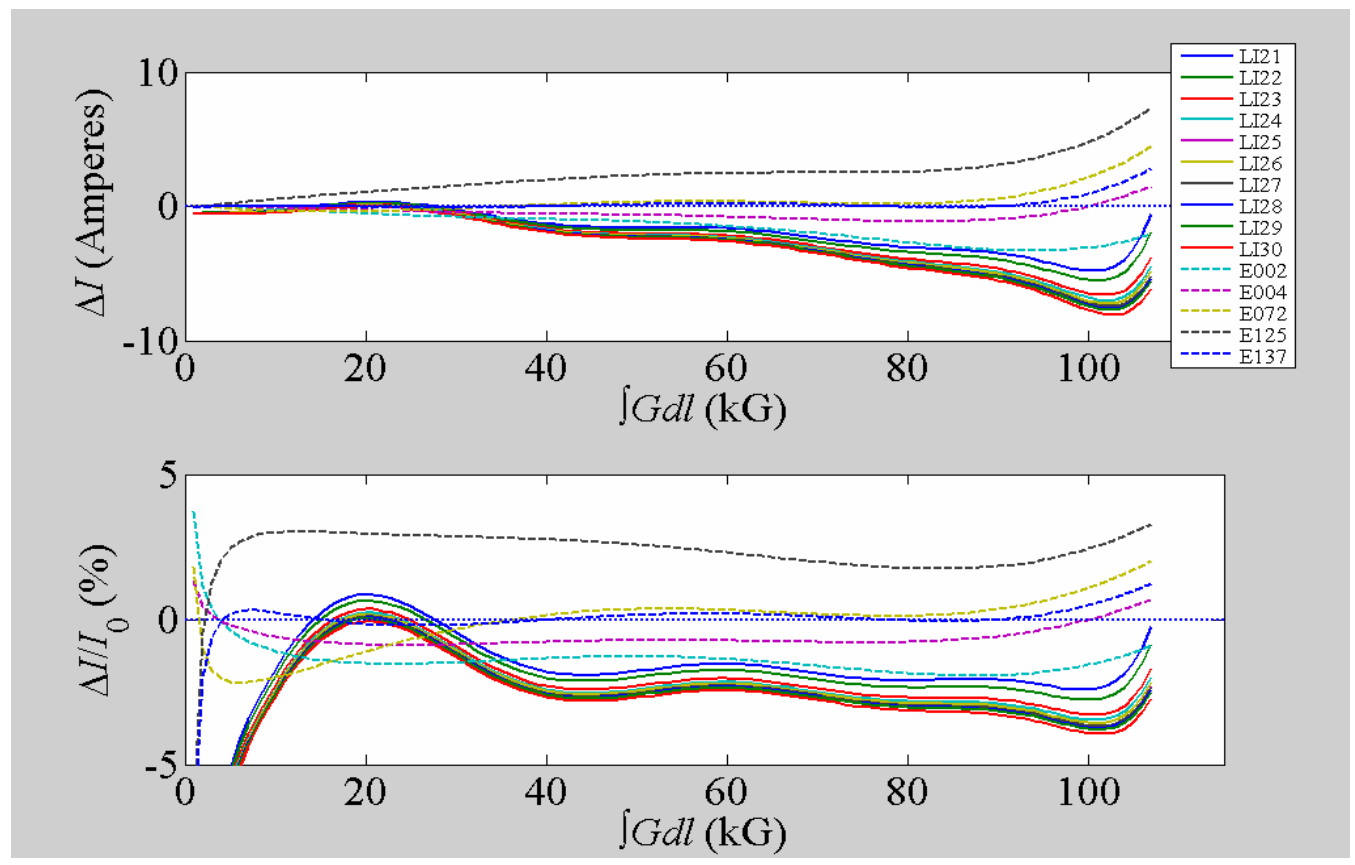

Figure 9. Absolute (top) and relative (bottom) current errors of the pre-2006 LI21-LI30 polynomials and the QE-002, 004, 072, 125, 137 polynomials, all with respect to the LCLS generic polynomial. The errors are reduced to $<2 \%$ (above $3 \mathrm{~A}$ ) by using specific polynomials for Q21201 through Q21601, and assuming QE-125 is an isolated anomaly. 
It is worth noting that the pre-2006 sector 21-30 polynomials show small scale variations from sector to sector (see Fig. 9), likely based on variations in the steel used, but not actual field measurements. These variations, however, are nearly insignificant compared to measured variations from magnet to magnet and will therefore not be retained.

Finally, the two sets of eight 'QE' magnets in sectors 22 and 23 will also run at $<20 \mathrm{~A}$ in LCLS mode, whereas in sector 24 and beyond the current levels will exceed $20 \mathrm{~A}$. Therefore, the bulk power supplies in sectors 22 and 23 will ideally be switched off (with disconnecting switches) in LCLS mode, while in sector 24 and beyond the bulk supplies will run at low current levels of 10-20 A, in addition to the individual currents from the booster supplies in these sectors. As of this date (Dec. 2006), the sector 22 and 23 bulk disconnecting switches are not fully incorporated into the LCLS plans.

Table 2. Field-to-current polynomial coefficients (see Eq. (2)) for the linac QE magnets. All polynomials are stored in the IVBU database slots, except the reversible Q21201, which uses both IVBU (for 'SLC') \& IVBD (for LCLS). Quadrupole locations with “even units" are 201, 401, 601, and 801, while “odd units” are 301, 501, 701, and 901. Only the odd polynomial coefficients $\left(p_{1}, p_{3}, p_{5}\right)$ are sign-flipped from even units to odd.

\begin{tabular}{|l|c|c|c|c|c|c|}
\hline quadrupole & $\begin{array}{c}\boldsymbol{p}_{\mathbf{0}} \\
{[\mathbf{A}]}\end{array}$ & $\begin{array}{c}\boldsymbol{p}_{\mathbf{1}} \\
{[\mathbf{A} / \mathbf{k G}]}\end{array}$ & $\begin{array}{c}\boldsymbol{p}_{\mathbf{2}} \\
{\left[\mathbf{A} / \mathbf{k G}^{\mathbf{2}}\right]}\end{array}$ & $\begin{array}{c}\boldsymbol{p}_{\mathbf{3}} \\
{\left[\mathbf{A} / \mathbf{k G}^{3}\right]}\end{array}$ & $\begin{array}{c}\boldsymbol{p}_{\mathbf{4}} \\
{\left[\mathbf{A} / \mathbf{k G}^{\mathbf{}}\right]}\end{array}$ & $\begin{array}{c}\boldsymbol{p}_{\mathbf{5}} \\
{\left[\mathbf{A} / \mathbf{k G}^{\mathbf{5}}\right]}\end{array}$ \\
\hline \hline Q21201 (IVBU) & -0.26462 & +1.7639 & $-2.1875 \mathrm{E}-3$ & $+1.6826 \mathrm{E}-4$ & $-3.3283 \mathrm{E}-6$ & $+2.0965 \mathrm{E}-8$ \\
\hline Q21201 (IVBD) & -0.26462 & -1.7639 & $-2.1875 \mathrm{E}-3$ & $-1.6826 \mathrm{E}-4$ & $-3.3283 \mathrm{E}-6$ & $-2.0965 \mathrm{E}-8$ \\
\hline Q21301 & -0.33584 & -1.8316 & $-6.4480 \mathrm{E}-3$ & $-2.3958 \mathrm{E}-4$ & $-3.7673 \mathrm{E}-6$ & $-2.1589 \mathrm{E}-8$ \\
\hline Q21401 & -0.29063 & +1.8225 & $-7.1109 \mathrm{E}-3$ & $+2.7003 \mathrm{E}-4$ & $-4.2159 \mathrm{E}-6$ & $+2.3483 \mathrm{E}-8$ \\
\hline Q21501 & -0.54638 & -1.9244 & $-7.4269 \mathrm{E}-3$ & $-2.5822 \mathrm{E}-4$ & $-4.0705 \mathrm{E}-6$ & $-2.3320 \mathrm{E}-8$ \\
\hline Q21601 & -0.51345 & +1.8870 & $-9.4876 \mathrm{E}-3$ & $+3.2612 \mathrm{E}-4$ & $-4.7654 \mathrm{E}-6$ & $+2.5527 \mathrm{E}-8$ \\
\hline all even units* & -0.35668 & +1.8318 & $-4.6026 \mathrm{E}-3$ & $+1.7106 \mathrm{E}-4$ & $-2.7875 \mathrm{E}-6$ & $+1.6809 \mathrm{E}-8$ \\
\hline all odd units** & -0.35668 & -1.8318 & $-4.6026 \mathrm{E}-3$ & $-1.7106 \mathrm{E}-4$ & $-2.7875 \mathrm{E}-6$ & $-1.6809 \mathrm{E}-8$ \\
\hline
\end{tabular}

* All magnet locations downstream of Q21601 with even unit numbers: 201, 401, 601, and 801.

** All magnet locations downstream of Q21601 with odd unit numbers: 301, 501, 701, and 901.

\section{References}

[1] The needed power supply regulation tolerances are based on a typical $200-\mu \mathrm{m}$ rms transverse quadrupole misalignment and the need to keep the trajectory stable to $<10 \%$ of its rms size. This explains why the needed static gradient error tolerances are much larger. See 2006 EPAC Conference Proceedings, pg. 151: http://accelconf.web.cern.ch/AccelConf/e06/PAPERS/MOPCH049.PDF). 